\title{
Comparison of vitamin D status in spring 2007 with spring 2008 - preliminary findings of the D-FINES study
}

\author{
A. L. Darling ${ }^{1}$, J. L. Berry ${ }^{2}$ and S. A. Lanham-New ${ }^{1}$ \\ ${ }^{1}$ Nutritional Sciences Division, Faculty of Health and Medical Sciences, University of Surrey, Guildford GU2 $7 H X$ \\ and ${ }^{2}$ Vitamin D Research Group, Department of Medicine, University of Manchester, Manchester M13 9PL, UK
}

Vitamin D status is dependent on UVB exposure as well as dietary intake of vitamin D. 25(OH)D is produced from 7-dehydrocholesterol in the skin's epidermis under the action of UVB rays. $25(\mathrm{OH}) \mathrm{D}$ status throughout the year is to some extent dependent on the UVB exposure in the preceding summer. In the South of England, summer 2006 was less cloudy with a higher number of hours sunshine per day ( $28 \%$ more), a higher mean temperature and less rainfall than summer 2007 . There has been little empirical investigation to the differential effects of weather on vitamin D status, but theoretically a cloudy, cooler and wet summer could be detrimental to vitamin D status, due to less UVB radiation being able to penetrate clouds and reach the skin, as well as the wearing of heavier clothing and more time spent indoors. Therefore, it was hypothesized that in spring 2008 women would have lower $25(\mathrm{OH}) \mathrm{D}$ than in spring 2007 due to lower production of $25(\mathrm{OH}) \mathrm{D}$ in the previous summer.

Two hundred and seventy-nine Caucasian and 86 South Asian pre- and post-menopausal women were assessed every season for serum $25(\mathrm{OH}) \mathrm{D}$, vitamin D intake and UVB exposure as part of the D-FINES study (June 2006-May 2007). Subjects were invited back for another serum 25(OH)D and diet assessment in spring 2008. All women who attended both spring appointments and had not started to take vitamin supplements after the main study ended were included in this analysis. A Wilcoxon signed ranks test (non-parametric data) was used to assess difference in $25(\mathrm{OH}) \mathrm{D}$ between the two paired spring measurements. As can be seen in Table 1 , this showed no significant difference between $25(\mathrm{OH}) \mathrm{D}$ in the two springs for all women, or any ethnic and menopausal subgroup. This research suggests that contrary to theoretical predictions, cloudy and wet summer weather may not necessarily translate into a detriment to vitamin D status later in the year. Indeed, there was no significant difference between vitamin D status in the spring following a cloudy than a non-cloudy summer. This finding is important as it allows simplification of sun exposure advice for achieving adequate vitamin D status.

Table 1. Mean and SD for serum 25(OH)D by group: spring $2007 v$. spring 2008

\begin{tabular}{|c|c|c|c|c|c|c|}
\hline \multirow[b]{2}{*}{ 25(OH)D (nmol/l) } & \multicolumn{2}{|c|}{ Spring 2007} & \multicolumn{2}{|c|}{ Spring 2008} & \multicolumn{2}{|c|}{ Wilcoxon signed rank test statistics } \\
\hline & Mean & $\mathrm{SD}$ & Mean & $\mathrm{SD}$ & $Z$ & $P$ \\
\hline Post-menopausal Caucasian & 40.52 & 16.24 & 43.42 & 16.95 & -0.523 & 0.601 \\
\hline Post-menopausal Asian & 15.23 & 0.55 & 16.90 & 1.85 & -1.069 & 0.285 \\
\hline All women & 41.56 & 17.06 & 41.63 & 18.00 & -0.751 & 0.452 \\
\hline All Caucasian & 43.87 & 16.09 & 44.15 & 17.04 & -0.348 & 0.728 \\
\hline All Asian & 19.65 & 8.85 & 17.68 & 2.17 & 0.000 & 1.000 \\
\hline
\end{tabular}

